\title{
Frecuencia de fascioliasis en ganado y de Fasciola hepatica en caracoles Lymnaeidae en el distrito de Huanca, Arequipa, Perú
}

\author{
Frequency of fascioliasis in cattle and Fasciola hepatica in Lymnaeidae snails \\ in the district of Huanca, Arequipa, Peru
}

\author{
Edel Valkan Chávez-Arce ${ }^{1.2}$, Guido Zumarán ${ }^{1}$
}

\section{RESUMEN}

\begin{abstract}
El objetivo del presente estudio fue determinar la frecuencia de fascioliasis en el ganado y factores asociados, así como la frecuencia de infección con fases larvarias en caracoles Lymnaeidae en la zona ganadera del distrito de Huanca en Arequipa, Perú. Se recolectaron 179 muestras de heces de bovinos, ovinos y equinos, y 561 caracoles Lymnaeidae. Los factores asociados se determinaron aplicando una encuesta epidemiológica por cada animal del ganado, a través de la entrevista a los propietarios. Se analizaron los huevos de Fasciola hepatica obtenidos de las muestras fecales, mediante la técnica de cuatro tamices modificada. Los caracoles $(n=484)$ fueron triturados en láminas portaobjetos, a fin de liberar y analizar las fases larvarias del trematodo, en tanto que, 77 caracoles se procesaron mediante la técnica de Pointier para la identificación taxonómica. La frecuencia de Fasciola hepatica en el ganado fue de 46.4\%, siendo mayor en ovinos y equinos, en animales que se alimentaban con alfalfa y pastos naturales, y en aquellos que abrevaban agua procedente de las acequias y el río. Se estableció como factor asociado a la fascioliasis la variable especie pecuaria. La frecuencia de infección en los Lymnaeidae fue 7.4\%. Los caracoles pertenecían al género Lymnaea.
\end{abstract}

Palabras clave: Fasciola hepatica, fascioliasis, factores asociados, Lymnaeidae, Lymnaea, Huanca

\footnotetext{
${ }^{1}$ Universidad Nacional de San Agustín de Arequipa

${ }^{2}$ E-mail: echavezar@unsa.edu.pe; https://orcid.org/0000-0002-5903-3777
}

Recibido: 21 de marzo de 2020

Aceptado para publicación: 15 de marzo de 2021

Publicado: 23 de junio de 2021

CLos autores. Este artículo es publicado por la Rev Inv Vet Perú de la Facultad de Medicina Veterinaria, Universidad Nacional Mayor de San Marcos. Este es un artículo de acceso abierto, distribuido bajo los términos de la licencia Creative Commons Atribución 4.0 Internacional (CC BY 4.0) [https:// creativecommons.org/licenses/by/4.0/deed.es] que permite el uso, distribución y reproducción en cualquier medio, siempre que la obra original sea debidamente citada de su fuente original 
The aim of this study was to determine the frequency of fascioliasis in cattle and its associated factors, as well as the frequency of infection with larval stages in Lymnaeidae snails in the livestock area of the Huanca district in Arequipa, Peru. In total, 179 samples of bovine, ovine and equine faeces, and 561 Lymnaeidae snails were collected. The associated factors were determined by applying an epidemiological survey for each livestock species, through the interview with the owners. Fasciola hepatica eggs obtained from faecal samples were analysed using the modified four-sieve technique. The snails $(n=484)$ were crushed on slides to release and analyse the larval stages of the trematode, while 77 snails were processed using the Pointier technique for taxonomic identification. The frequency of Fasciola hepatica in cattle was $46.4 \%$, being higher in sheep and horses, in animals that were fed with alfalfa and natural pastures, and in those that drank water from the ditches and the river. The variable livestock species was established as a factor associated with fascioliasis. The frequency of infection in the Lymnaeidae was $7.4 \%$. The snails belonged to the genus Lymnaea.

Key words: Fasciola hepatica, fascioliasis, associated factors, Lymnaeidae, Lymnaea, Huanca

\section{INTRODUCCIÓN}

La fascioliasis o distomatosis causada por el trematodo Fasciola hepatica, es una zoonosis de gran importancia en el mundo que ocasiona sobre todo patologías hepatobiliares (Espinoza et al., 2010). El parásito tiene de huésped intermediario a los caracoles del género Lymnaea (Familia: Lymnaeidae) y como hospederos definitivos una gran variedad de mamíferos herbívoros, afectando también al humano (Carrada-Bravo, 2007; Pujadas et al., 2015; Vázquez y Sánchez, 2015). Los animales se infectan en el pastoreo o al beber agua contaminada con estados inmaduros del parásito (Carrada-Bravo, 2007; López et al., 2017).

La región de Ayacucho, Perú, es endémica a la Fasciola, encontrándose prevalencias de infección bovina y ovina de 47.6 y $52.1 \%$, respectivamente (Ticona et al., 2010); asimismo, las regiones de Apurímac, Arequipa, y La Libertad presentan preva- lencias elevadas de fascioliasis en bovinos, ovinos y equinos (Merino y Valderrama, 2017; Velásquez, 2017; Jara et al., 2018). Prevalencias elevadas igualmente se presentan en otros países de América Latina (Alpízar et al., 2013; Giraldo et al., 2016; Soca-Pérez et al., 2016).

Se reportan grandes pérdidas económicas causadas por la distomatosis, tanto en países africanos (Berthe et al., 2017; Gebeye et al., 2017; Jaja et al., 2017; Liba et al., 2017; Meaza et al., 2017), como en países latinoamericanos como Costa Rica (Rojas y Cartín, 2016) y Cuba (Palacio et al., 2019). En forma similar, los sistemas pecuarios de producción del país se encuentran afectados, con pérdidas que rodean los 50 millones de dólares anuales, siendo la principal causa el decomiso de vísceras como hígados infectados con fasciolas jóvenes o adultas, la reducción de la ganancia de peso, tasas de preñez decrecientes y la producción mermada de leche y carne (Espinoza et al., 2010; López et al., 2017). 
Estudios epidemiológicos sobre los hospederos intermediarios de $F$. hepatica indican prevalencias de infección natural del 12 y $27 \%$ en Lymnaea columella y L. viatrix, respectivamente, en Cajamarca, Trujillo, Huánuco, Lima y Ucayali (Larrea et al., 2007). Asimismo, la frecuencia en Cusco asciende al 36 y $46 \%$ en caracoles de Pseudosuccinea columella recolectados entre 4200 y $4500 \mathrm{msnm}$, y $48 \%$ en individuos de L. viatrix muestreados entre 4000 y 4200 msnm (Londoñe et al., 2009).

La creciente y persistente presencia de $F$. hepatica en el territorio peruano, es indicativo de la ausencia de programas eficientes de prevención y control del parásito, los cuales deben estar orientados a mejorar la forma de crianza del ganado, la estratégica aplicación de tratamientos veterinarios y de la buena ejecución de los sistemas de gestión sanitaria en el manejo de las heces, así como de los cuerpos de agua, y a desarrollar una cultura consciente y responsable en las comunidades (Espinoza et al., 2010).

La Gerencia Regional de Salud de Arequipa emitió un informe en 2014 a través del Análisis de Situación de Salud de los casos de distomatosis humana encontrados hasta dicho año, siendo la provincia de Caylloma la localidad más afectada (GERESA, 2014). A la fecha, aún persiste el desconocimiento epidemiológico de esta zoonosis en la zona y, por ello, el objetivo del presente estudio fue determinar la frecuencia de la fascioliasis animal en el ganado, sus factores asociados y la frecuencia de infección por $F$. hepatica en caracoles Lymnaeidae.

\section{Materiales y Métodos}

\section{Área de Estudio}

La investigación se realizó entre agosto y setiembre de 2018 en las comunidades de Huanca, San Basilio y Malata, distrito de Huanca, provincia de Caylloma, Arequipa,
Perú. El distrito forma parte de la vertiente occidental de los Andes del Sur del Perú y se encuentra a una altitud de $3080 \mathrm{msnm}$. El clima es semiseco y frío, de baja humedad relativa, temperatura media anual de $15.2^{\circ} \mathrm{C}$ y precipitaciones anual entre 300 y $500 \mathrm{~mm}$. El territorio está irrigado por los ríos Quilca, Siguas, Vitor y Chili y cuenta con aguas subterráneas empleadas en la agricultura. Huanca está atravesada por dos afluentes del río Siguas, Lihualla y Pichirijma. Su estructura agraria se compone de pequeñas parcelas de forma irregular, debido al accidentado relieve y las pendientes profundas que representan el $70 \%$ del total del suelo. El agua utilizada para el riego proviene del glaciar Ampato, que discurre por gravedad hasta los andenes. La ganadería es de tipo extensiva, en su mayoría bovina, seguida de ovina, equina y escasamente porcina (Trujillo, 2015; Trujillo y Lajo, 2015).

\section{Muestras}

El trabajo se realizó en ganaderías extensivas, donde los animales no son marcados ni identificados adecuadamente, de allí que las muestras fecales de bovinos, ovinos y equinos se recolectaron por conveniencia, recorriendo cerca de $20 \mathrm{~km}$ entre las áreas pecuarias de las comunidades de Malata, San Basilio y el pueblo de Huanca (comunidad principal del distrito), con la intención de tener encuentros casuales con el ganado y sus propietarios (Otzen y Manterola, 2017). Las muestras fueron colectadas inmediatamente después de la emisión fecal $(\mathrm{n}=179)$ según el Manual Veterinario de Toma y Envío de Muestras de PANAFTOSA (2017) modificado. Un puñado de la parte superficial de las bostas fueron levantadas del suelo con una bolsa de polietileno, y luego se fijaron en solución formol al 7\%. En forma paralela, se encuestó a los dueños a través de una ficha epidemiológica en función de las siguientes variables: comunidad (Huanca, Malata, San Basilio) especie pecuaria (bovino, equino, ovino), alimento de consumo (alfalfa, pastos naturales, concentrado) y fuente de agua (acequia, río, pozo). 

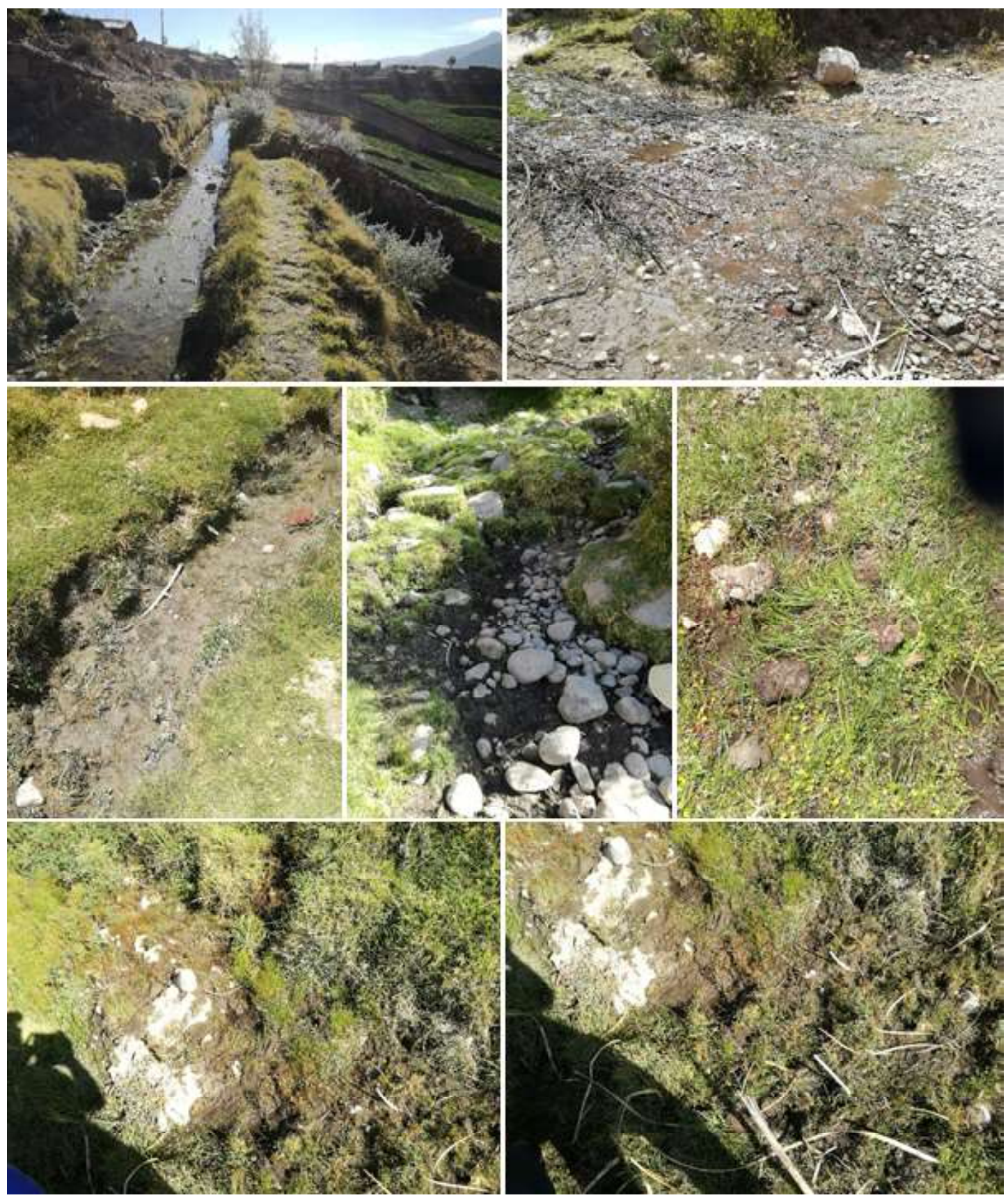

Figura 1. Zonas potenciales para el desarrollo de los caracoles Lymnaeidae como acequias, riachuelos, suelos lodosos y pequeños cuerpos de agua empozados

Para la recolección de caracoles Lymnaeidae, se identificaron las áreas adecuadas para su desarrollo, como la ribera de pequeños ríos, bajo las piedras de los cuerpos de agua, en charcos, agua empozada, acequias, arroyos y fango (Figura 1). Considerando lo anterior, el muestreo se realizó al- rededor de las coordenadas de los siguientes puntos: Comunidad de Huanca $\left[\mathrm{P}_{1}=16^{\circ} 02^{\prime}\right.$ $16^{\prime}$ ' $\mathrm{S}-71^{\circ} 52^{\prime} 24^{\prime \prime} \mathrm{W}$ (fango formado cerca de un camino empinado); $\mathrm{P}_{2}=16^{\circ} 01^{\prime}$ 55" $\mathrm{S}-71^{\circ} 52^{\prime} 28^{\prime \prime} \mathrm{W}$ (arroyo pedregoso); $\mathrm{P}_{3}=16^{\circ}$ 02 ' 37' $\mathrm{S}-71^{\circ} 52^{\prime} 54$ " W (canal de regadío) $]$ y comunidad de Malata $\left[\mathrm{P}_{4}=16^{\circ} 02^{\prime} 32^{\prime \prime}\right.$ 


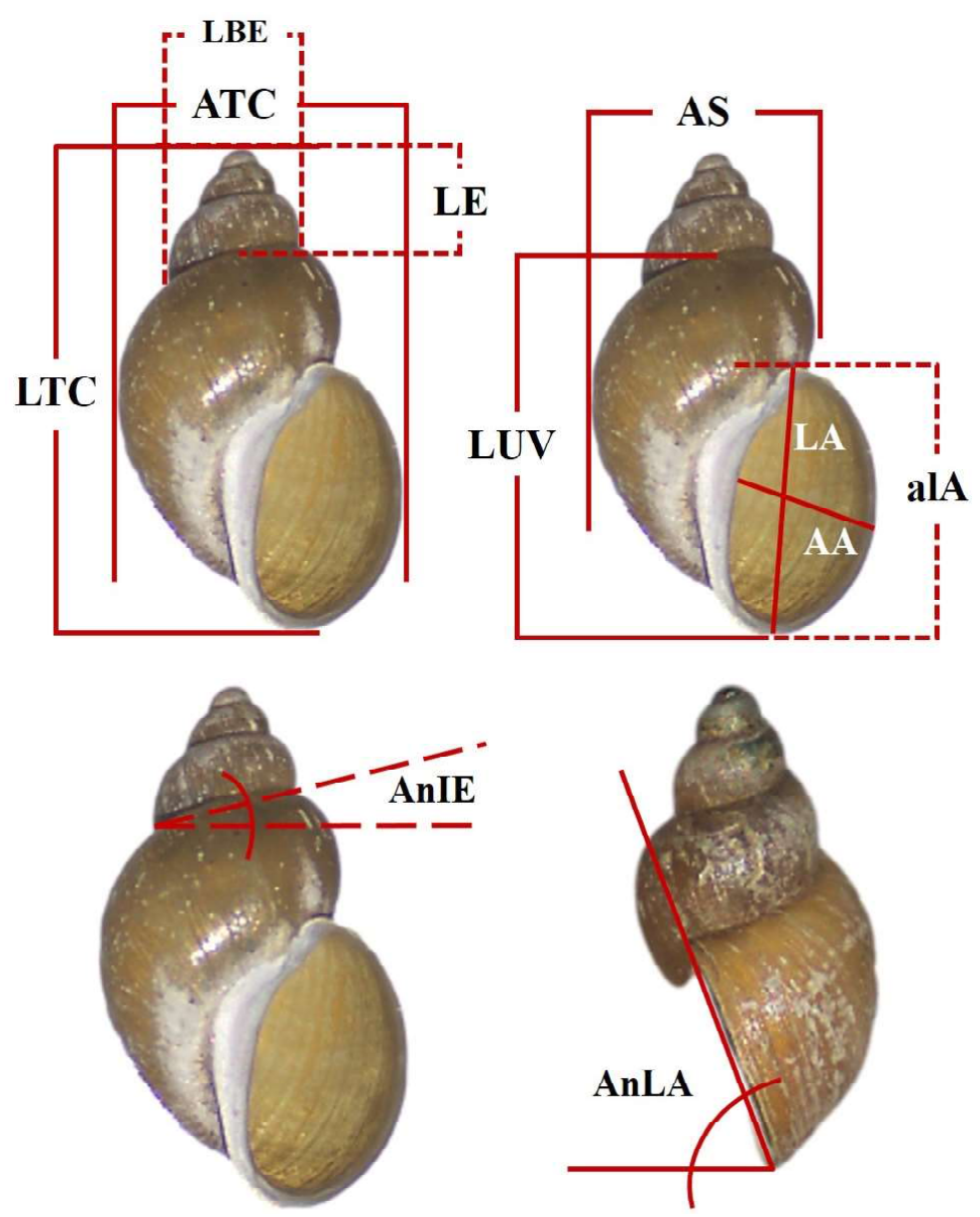

Figura 2. Mediciones consideradas en la morfometría de las conchillas de los caracoles Lymnaeidae. LTC: largo total de la conchilla; ATC: ancho total de la conchilla; LA: largo de la abertura; AA: ancho de la abertura; alA: altura de la abertura; AS: ancho superior; LE: largo de la espira; LBE: longitud de la base de la espira; LUV: longitud de la última vuelta; LTC-alA: largo restante de la conchilla; AnIE: ángulo de inclinación de la espira; AnLA: ángulo lateral de la abertura.

$\mathrm{S}-71^{\circ} 51^{\prime} 43^{\prime \prime} \mathrm{W}$ (fango formado cerca a la entrada de Malata); $\mathrm{P}_{5}=160231 \mathrm{~S}-71^{\circ} 51^{\prime}$ 28 " W (canal de regadío)]. Se recolectaron manualmente 561 caracoles, considerando las características conquiológicas reconocibles en campo de un lymneido, como la forma oval de la conchilla, suturas profundas, el color pardo y, principalmente, la abertura en posición dextrógira (Carvalho et al., 2014; Vázquez y Sánchez, 2015). Los lymneidos fueron llevados al laboratorio en frascos de plástico de boca ancha conteniendo algodón hidrófilo humedecido con agua de su hábitat (Iturbe y Muñiz, 2012). 


\section{Análisis de Laboratorio}

Las muestras fueron evaluadas en el Laboratorio de Entomología y Protección Vegetal de la Escuela de Biología de la Universidad Nacional de San Agustín de Arequipa. Las heces se procesaron mediante la Técnica de Cuatro Tamices de Girão y Ueno (1985) modificada para el presente estudio, utilizando tres tamices de acero inoxidable en vez de cuatro, pero de métrica semejante, con aberturas de malla de $150,75 \mathrm{y}$ $63 \mu \mathrm{m}$. Primeramente, las heces fueron homogenizadas con la solución fijadora, se apilaron los tamices colocando en la parte baja el de $63 \mu \mathrm{m}$ y en la parte superior el de $150 \mu \mathrm{m}$. Se tamizó $50 \mathrm{~g}$ de cada muestra moviendo bien los tamices con bastante agua a chorro. Este paso se repitió sin el tamiz de $150 \mu \mathrm{m}$ y nuevamente quitando el de $75 \mu \mathrm{m}$, permaneciendo solo el más fino de $63 \mu \mathrm{m}$. El sobrenadante fue eliminado y lo que quedó se transfirió en su totalidad a una placa Petri, a la cual se agregó de 3 a 4 gotas de azul de metileno que sirvió como fondo de contraste. El criterio de positividad de fascioliasis fue por el hallazgo de huevos de $F$. hepatica con base a las descripciones de Bedoya et al. (2012) y Beltrán et al. (2014).

La evaluación de los caracoles $(n=484)$ fue mediante el aplastamiento individual en láminas portaobjetos con una gota de suero fisiológico. Los preparados fueron triturados mediante el uso de pinzas (Londoñe et al., 2009; Giménez et al., 2014). El criterio de positividad a la infección fue por el hallazgo de redias y cercarias de $F$. hepatica, según los criterios morfológicos descritos por López et al. (2008).

Para la identificación taxonómica de los moluscos, se procesaron 77 ejemplares según la técnica de Pointier, tomada de Tomassi et al. (2015). Para esto, los caracoles fueron lavados con agua corriente a fin de eliminar las impurezas, luego se sumergieron en agua a $70{ }^{\circ} \mathrm{C}$ por $30 \mathrm{~s}$, de allí en agua fría y en solución alcohol a $70^{\circ}$ durante $24 \mathrm{~h}$. Posteriormente, con pinzas y agujas se retiró la masa blanda y fue conservada en solución etanol a $70^{\circ}$ en viales de $15 \mathrm{ml}$. En el estudio conquiológico se utilizó el software ZEN v. 2.3 lite y un estereomicroscopio. Se describieron las siguientes características: forma de la conchilla, color de la conchilla, aspecto de las suturas, número de vueltas y posición de la abertura (Carvalho et al., 2014). En la morfometría se midió (Figura 2) el largo total de la conchilla (LTC), ancho total de la conchilla (ATC), largo de la abertura (LA), ancho de la abertura (AA), altura de la abertura (alA), ancho superior (AS), largo de la espira (LE); longitud de la base de la espira (LBE), longitud de la última vuelta (LUV), largo restante de la conchilla (LTC-alA), ángulo de inclinación de la espira (AnIE) y ángulo lateral de la abertura (AnLA). Así mismo, fueron calculadas las proporciones de LUV/LTC, LE/LTC, alA/LTC, alA/LUV, ATC/LTC, AA/LA, AA/ATC, AS/ATC, LBE/ ATC y LBE/AS, según lo considerado por Damiani y Álvarez (2005), Núñez (2011), Vázquez y Sánchez (2015), Bargues et al. (2016), Dar et al. (2016), y De Lucía y Gutiérrez (2017).

\section{Análisis Estadístico}

Los datos fueron analizados utilizando los softwares estadísticos IBM SPSS Statistics v. 25 y Epidat 4.2. La frecuencia de infección en el ganado y en los caracoles se determinó mediante el cálculo de las proporciones porcentuales de animales y moluscos positivos a huevos y larvas de $F$. hepatica, respectivamente, acompañados de sus intervalos de confianza (IC). De la merística de las conchillas, se calculó como medida de posición la media aritmética, acompañada de las medidas de dispersión de valor máximo y mínimo, rango, error estándar, desviación estándar y para mostrar la precisión de las medias los IC al 95\% (Milton, 2007; Celis de la Rosa, 2014). Para la identificación de los factores asociados a la fascioliasis, se usó la prueba de regresión logística siguiendo una técnica backward (Acuña et al., 2012), a fin de calcular los OR ajustados (ORa). Se usó un nivel de significación del 5\% (Cerda et al., 2013; Dagnino, 2014). 
Cuadro 1. Frecuencia de Fasciola hepatica en el ganado del distrito de Huanca, provincia de Caylloma, Arequipa (2018)

\begin{tabular}{|c|c|c|c|c|}
\hline \multirow{2}{*}{ Variables } & \multirow{2}{*}{ Total } & \multicolumn{3}{|c|}{ Positivos } \\
\hline & & $\mathrm{n}$ & $\%$ & IC 95\% \\
\hline \multicolumn{5}{|l|}{ Comunidad } \\
\hline Huanca & 68 & 33 & 48.5 & $36.7-60.4$ \\
\hline Malata & 36 & 13 & 36.1 & $20.4-51.8$ \\
\hline San Basilio & 75 & 37 & 49.3 & $38.0-60.6$ \\
\hline \multicolumn{5}{|l|}{ Especie pecuaria } \\
\hline Bovinos & 137 & 58 & 42.3 & $34.1-50.6$ \\
\hline Ovinos & 25 & 16 & 64.0 & $45.2-82.8$ \\
\hline Equinos & 17 & 9 & 52.9 & 29.2-76.7 \\
\hline \multicolumn{5}{|l|}{ Alimento de consumo } \\
\hline Alfalfa y pastos naturales & 160 & 75 & 46.9 & $39.1-54.6$ \\
\hline $\begin{array}{l}\text { Alfalfa, pastos naturales y } \\
\text { concentrado }\end{array}$ & 19 & 8 & 42.1 & $19.9-64.3$ \\
\hline \multicolumn{5}{|l|}{ Fuente de agua } \\
\hline Acequia y pozo & 22 & 10 & 45.5 & $24.6-66.3$ \\
\hline Acequia y río & 157 & 73 & 46.5 & $38.7-54.3$ \\
\hline Total & 179 & 83 & 46.4 & $39.1-53.7$ \\
\hline
\end{tabular}

IC: Intervalo de confianza

\section{Resultados}

La frecuencia de fascioliasis en el ganado fue de $46.4 \%$ ( $\mathrm{IC}=39.1-53.7$ ), siendo mayor en la localidad de San Basilio con $49.3 \%$ ( $\mathrm{IC}=38.0-60.6)$, en los ovinos $64.0 \%$ ( $\mathrm{IC}=45.2-82.8)$, en el ganado que consumía alfalfa y pastos naturales $46.9 \%$ (IC=39.154.6), y en los animales que abrevaban agua de las acequias y el río 46.5\% ( $\mathrm{IC}=38.7-54.3$ ), según se muestra en el Cuadro 1. La prueba de regresión logística estableció que la variable especie pecuaria $(\mathrm{p}=0.046<0.05 ; \mathrm{IC}=1.0$ $4.2>1)$ constituyó un factor asociado a la infección por $F$. hepatica en el ganado del distrito de Huanca, mostrando que los equinos y ovinos tenían 2.1 veces más probabilidad de desarrollar fascioliasis (Cuadro 2).

Los huevos de Fasciola eran ovalados, operculados por un extremo y de típico color amarillo que fácilmente contrastaba con el colorante (Figura 3). La frecuencia de estados larvales de $F$. hepatica en los caracoles fue de 7.4\% ( $\mathrm{IC}=5.1-9.8)$, siendo mayor en la comunidad de Huanca, alcanzando un 9.8\% ( $\mathrm{IC}=5.2-14.3)$; sin embargo, en San Basilio no se encontró a los moluscos (Cuadro 3). Las redias presentaban una ventosa oral, saco digestivo y proyecciones laterales a lo largo del cuerpo; las cercarias poseían una ventosa oral, acetábulo, abundantes glándulas cistógenas y carecían de manchas oculares (Figura 4). 


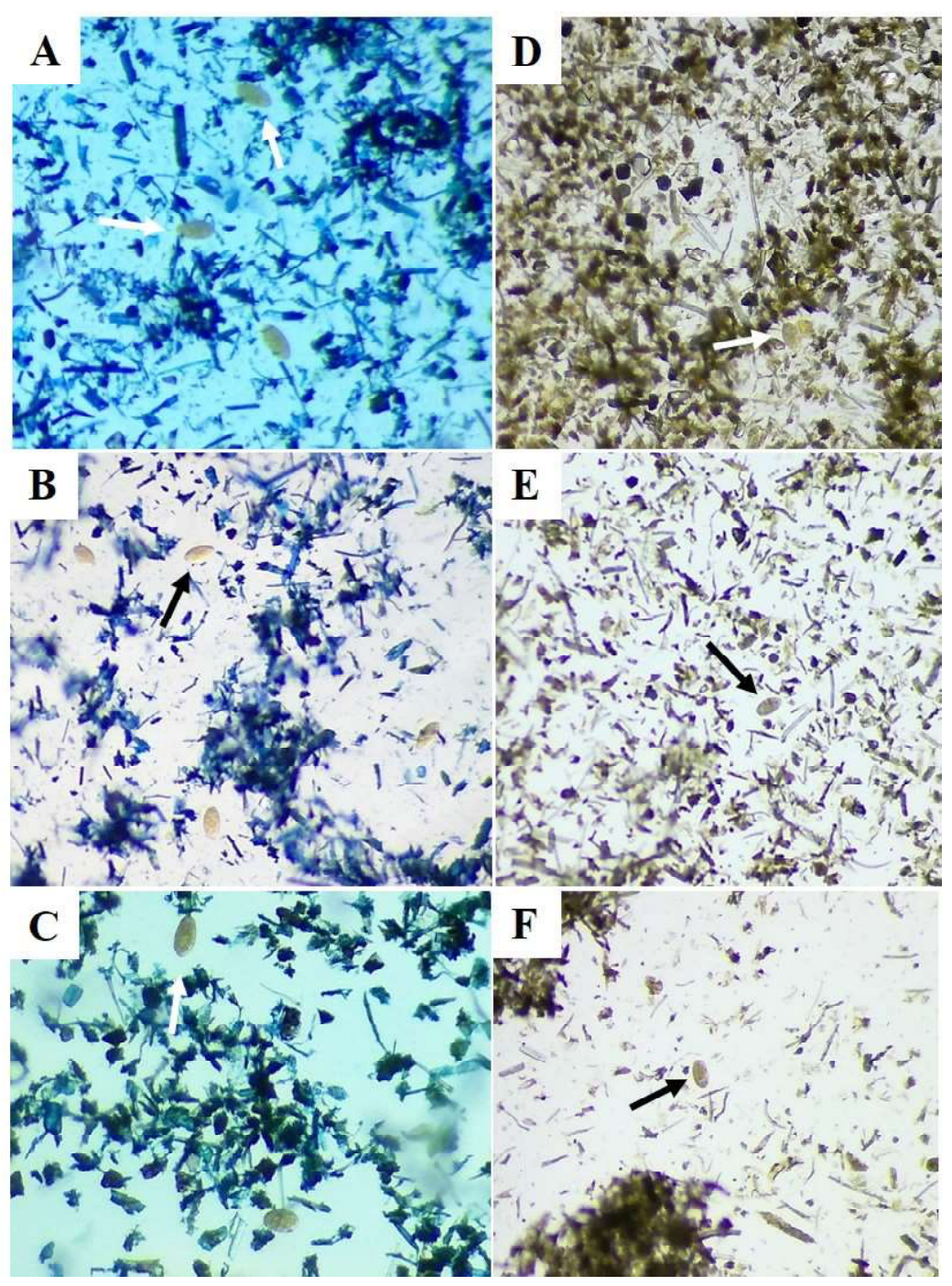

Figura 3. Huevos de Fasciola hepatica hallados en heces del ganado bovino, ovino y equino del distrito de Huanca, provincia de Caylloma, Arequipa (2018) (flechas blancas y negras). A-C: en azul de metileno; D-F: sin tinción. 2x y 4x

Se observaron las siguientes características conquiológicas: conchilla oval elongada, color marrón, algunos blanquecinos y otros mixtos, ápice de la espira obtuso, giros (vueltas) hacia la derecha, suturas marcadas y con 4 o 5 vueltas y media. Abertura dextrógira, ovalada, con pliegue columelar y fino peristoma. El largo total promedio fue $5.21 \pm 0.11 \mathrm{~mm}$. Los ejemplares más pequeños midieron $3.09 \mathrm{~mm}$ y los de mayor tama- ño $7.05 \mathrm{~mm}$. Ancho total promedio de $2.82 \pm 0.05 \mathrm{~mm}$, la longitud de la última vuelta representó 3/4 del LTC (78\%), la altura de la abertura la mitad del LTC (52\%) y un poco más respecto a la LUV, alcanzando casi las $3 / 4$ partes $(67 \%)$. El ATC medía un poco más de la mitad del LTC (54\%), el AA fue de igual forma respecto al LA (54\%) y al ATC (53\%); sin embargo, el AS representó un poco más de los 3/4 del ATC. La LBE fue propor- 

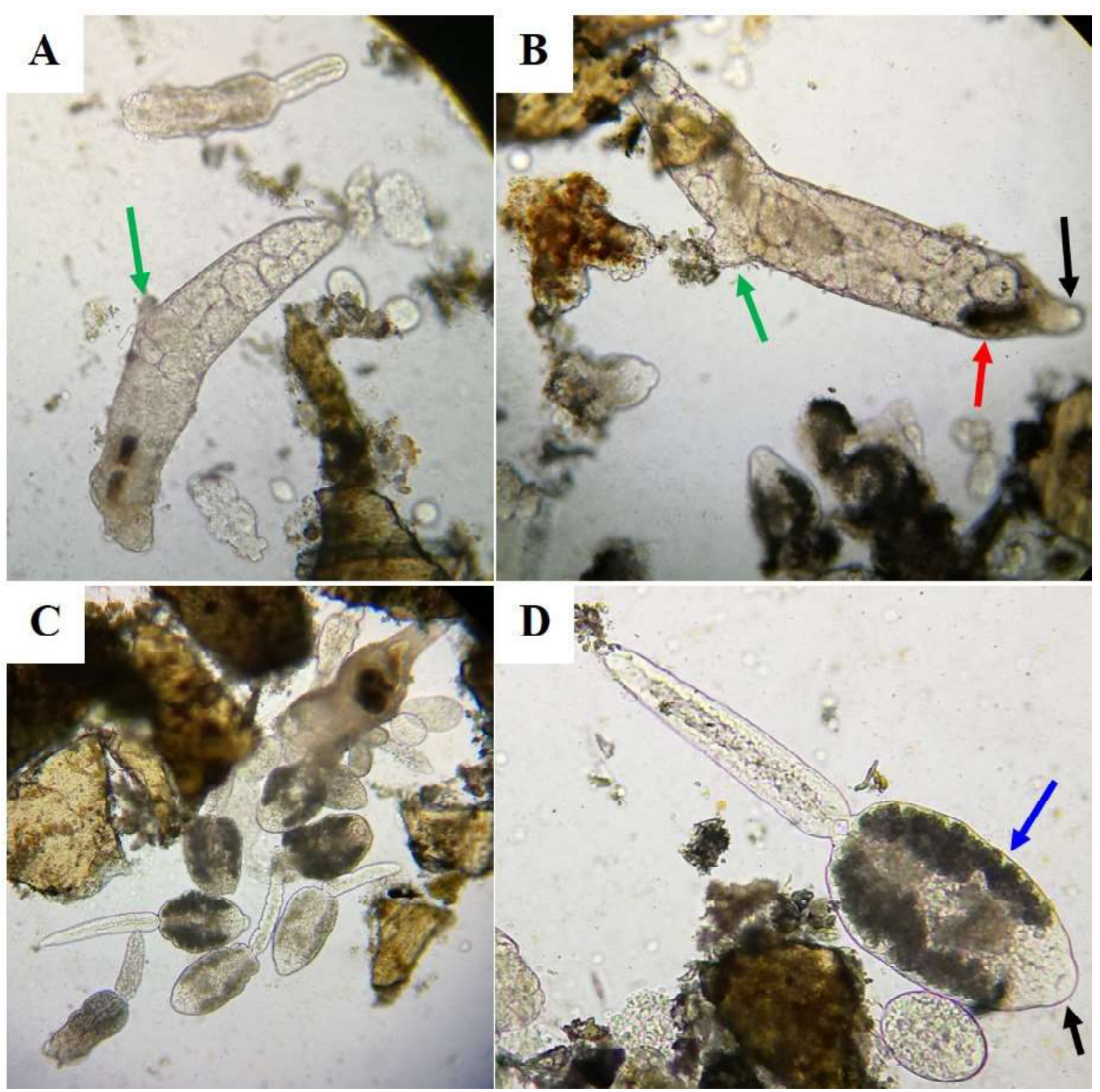

Figura 4. Estados larvales de Fasciola hepatica, hallados en los caracoles Lymnaeidae del distrito de Huanca, provincia de Caylloma, Arequipa (2018). A-B: Redias; C-D: Cercarias; Flecha negra: Ventosa oral; Flecha verde: Proyección lateral; Flecha roja: Saco digestivo; Flecha azul: Glándulas cistógenas. 2x y 4x

cional a la mitad del ATC (52\%) y un poco más respecto al AS (Cuadro 4). La inclinación de la espira formó un ángulo de $7.41 \pm 0.37^{\circ}$ y en vista lateral $70.28 \pm 0.36^{\circ}$. En base a lo descrito, se determinó que los caracoles pertenecían al género Lymnaea de la Familia Lymneidae (Figura 5).

\section{Discusión}

Se encontró una frecuencia de fascioliasis en el ganado del $46.4 \%$, por lo que se podría considerar al distrito de Huanca como una zona mesoendémica de la fascioliasis animal (Valderrama, 2016); sin embargo, la 
Cuadro 2. Factores asociados a la fascioliasis en el ganado del distrito de Huanca, provincia de Caylloma, Arequipa (2018)

\begin{tabular}{lccc}
\hline \multirow{2}{*}{ Variables } & \multicolumn{2}{c}{$*$ ORa } & \multirow{2}{*}{ Valor p } \\
\cline { 2 - 3 } & $\begin{array}{c}\text { Estimación de } \\
\text { punto }\end{array}$ & IC 95\% & \\
\hline Especie pecuaria $\dagger$ & 2.1 & $1.0-4.2$ & 0.046 \\
Alimento de consumo & 1.3 & $0.5-3.6$ & 0.563 \\
Fuente de agua & 1.2 & $0.5-3.0$ & 0.691 \\
\hline
\end{tabular}

*ORa : Odds ratio ajustado calculado mediante análisis de regresión logística.

† Ovino y equino vs bovino

IC: Intervalo de confianza

Cuadro 3. Frecuencia de Fasciola hepatica en caracoles Lymnaeidae del distrito de Huanca, provincia de Caylloma, Arequipa (2018)

\begin{tabular}{lcccc}
\hline \multirow{2}{*}{ Comunidades } & Total & \multicolumn{3}{c}{ Positivos } \\
\cline { 3 - 5 } & & $\mathrm{n}$ & $\%$ & $\mathrm{IC} \mathrm{95 \%}$ \\
\hline Huanca & 164 & 16 & 9.8 & $5.2-14.3$ \\
Malata & 320 & 20 & 6.3 & $3.6-8.9$ \\
San Basilio & 0 & 0 & 0.0 & - \\
\hline Total & 484 & 36 & 7.4 & $5.1-9.8$ \\
\hline
\end{tabular}

IC: Intervalo de confianza

cifra real fue probablemente mayor, dado que el diagnóstico fue mediante la observación de huevos de $F$. hepatica obtenidos en el tamizado de muestras fecales, los cuales están presentes únicamente en la fase crónica de la infección (López et al., 2017). Por otra parte, actualmente se dispone de otros métodos o técnicas de diagnóstico de mayor precisión, tales como los inmunológicos ELISA (Alzamora-Gonzales et al., 2016) y Western Blot, que permiten detectar antígenos y anticuerpos coprológicos y serológicos, ade- más de las técnicas moleculares como PCR para identificar presencia de material genético de F. hepatica (Gomez-Puerta et al., 2019). Esto se puede evidenciar en el estudio de Jara et al. (2018), en el que compararon sus resultados de prevalencias por varios métodos de diagnóstico, llegando a obtener una seroprevalencia de $61.8 \%$ en ovinos y $86.8 \%$ en bovinos con la técnica de Western blot, cifra superior al 42.3 y $62.4 \%$ obtenidas mediante las técnicas coprológicas Kato-Katz cualitativo y Ritchie para estas especies. 
Cuadro 4. Medidas morfométricas de las conchillas de los caracoles Lymnaeidae colectados en el distrito de Huanca, provincia de Caylloma, Arequipa (2018)

\begin{tabular}{lccccc}
\hline Medidas $(\mathrm{mm})$ & $\overline{\mathrm{x}} \pm \mathrm{SE}$ & $\mathrm{DE}$ & Min. - Max. & Rango & IC $95 \%$ \\
\hline LTC & $5.21 \pm 0.11$ & 0.96 & $3.09-7.05$ & 3.95 & $5.00-5.43$ \\
ATC & $2.82 \pm 0.05$ & 0.47 & $1.76-3.72$ & 1.96 & $2.72-2.93$ \\
LA & $2.75 \pm 0.06$ & 0.49 & $1.70-3.78$ & 2.08 & $2.64-2.85$ \\
AA & $1.49 \pm 0.03$ & 0.28 & $0.85-2.20$ & 1.35 & $1.43-1.56$ \\
alA & $2.72 \pm 0.06$ & 0.50 & $1.66-4.21$ & 2.55 & $2.61-2.83$ \\
AS & $2.43 \pm 0.05$ & 0.42 & $1.08-3.22$ & 2.14 & $2.34-2.52$ \\
LE & $1.17 \pm 0.04$ & 0.34 & $0.03-1.83$ & 1.80 & $1.09-1.25$ \\
LBE & $1.46 \pm 0.03$ & 0.26 & $1.00-2.40$ & 1.40 & $1.40-1.52$ \\
LUV & $4.04 \pm 0.08$ & 0.71 & $2.35-5.48$ & 3.13 & $3.88-4.20$ \\
LTC-alA & $2.49 \pm 0.06$ & 0.57 & $1.14-3.52$ & 2.38 & $2.37-2.62$ \\
AnIE $\left(^{\circ}\right)$ & $7.41 \pm 0.37$ & 3.25 & $0.81-14.12$ & 13.32 & $6.68-8.14$ \\
AnLA $\left({ }^{\circ}\right)$ & $70.28 \pm 0.36$ & 3.15 & $62.72-81.64$ & 18.92 & $69.57-70.99$ \\
LUV/LTC & $0.78 \pm 0.00$ & 0.04 & $0.66-0.99$ & 0.34 & $0.77-0.79$ \\
LE/LTC & $0.22 \pm 0.00$ & 0.04 & $0.01-0.34$ & 0.34 & $0.21-0.23$ \\
alA/LTC & $0.52 \pm 0.01$ & 0.05 & $0.42-0.76$ & 0.42 & $0.51-0.53$ \\
alA/LUV & $0.67 \pm 0.1$ & 0.05 & $0.48-1.00$ & 0.52 & $0.66-0.69$ \\
ATC/LTC & $0.54 \pm 0.00$ & 0.03 & $0.47-0.65$ & 0.17 & $0.54-0.55$ \\
AA/LA & $0.54 \pm 0.00$ & 0.03 & $0.47-0.64$ & 0.17 & $0.54-0.55$ \\
AA/ATC & $0.53 \pm 0.01$ & 0.05 & $0.46-0.90$ & 0.44 & $0.52-0.54$ \\
AS/ATC & $0.86 \pm 0.01$ & 0.06 & $0.43-1.08$ & 0.65 & $0.85-0.88$ \\
LBE/ATC & $0.52 \pm 0.01$ & 0.04 & $0.46-0.78$ & 0.33 & $0.51-0.53$ \\
LBE/AS & $0.61 \pm 0.01$ & 0.07 & $0.54-1.13$ & 0.59 & $0.59-0.62$ \\
\hline
\end{tabular}

x̄: Media aritmética. SE: Error estándar. DE: Desviación estándar. Min-Max: Mínimo y máximo. IC: Intervalo de confianza

LTC: largo total de la conchilla; ATC: ancho total de la conchilla; LA: largo de la abertura; AA: ancho de la abertura; alA: alto de la abertura; AS: ancho superior; LE: largo de la espira; LBE: longitud de la base de la espira; LUV: longitud de la última vuelta; LTC-alA: largo restante de la conchilla; AnIE: ángulo de inclinación de la espira; AnLE: ángulo lateral de la abertura. $\left({ }^{\circ}\right)$. Grados

Los ovinos y equinos mostraron mayor presencia de fascioliasis, coincidiendo con el análisis de Regresión logística, que estableció a la especie pecuaria como el único factor asociado a la zoonosis, teniendo los equinos y los ovinos 2.1 veces más probabilidad de desarrollar distomatosis hepática. Sin embargo, la literatura menciona otro tipo de resultados; así, Ticona et al. (2010) reportaron prevalencias de $39.1 \%$ de fascioliasis en ovinos y de $35.7 \%$ en bovinos de Ayacucho, mientras que Jara et al. (2018) estimaron $61.8 \%$ para ovinos y $86.8 \%$ para bovinos en La Libertad, Perú. 


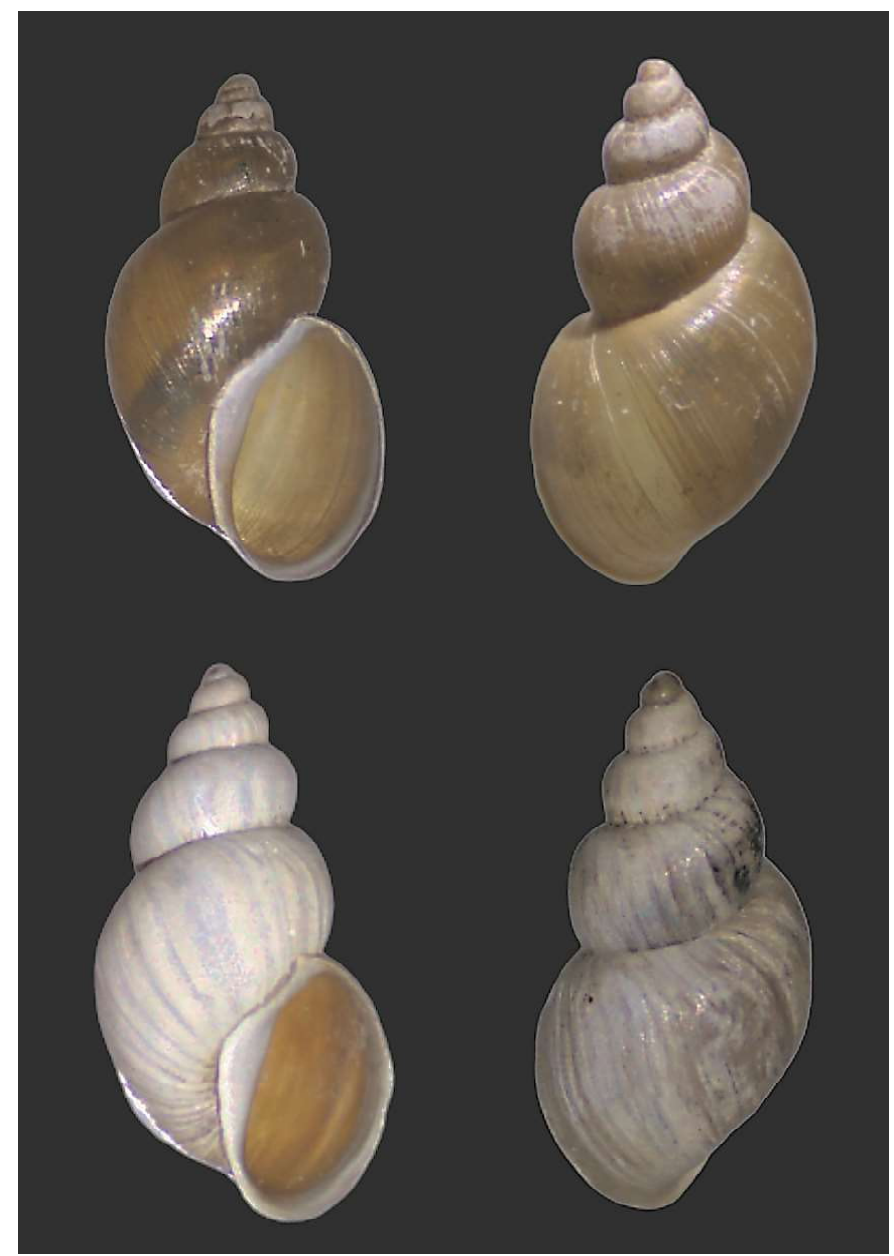

Figura 5. Vista ventral y dorsal de ejemplares Lymnaeidae recolectados en el distrito de Huanca, provincia de Caylloma, Arequipa (2018)

La frecuencia de infección fue semejante en las comunidades de Huanca y San Basilio y un poco menor en Malata. Esta diferencia probablemente se deba a los factores ambientales propios de cada zona, como la calidad del agua en relación con el $\mathrm{pH}$, temperatura, salinidad y oxígeno disuelto, así como la humedad ambiental y el tipo de suelo (Carrada-Bravo y Escamilla, 2005; CarradaBravo, 2007). Otras causas podrían ser las posibles prácticas inadecuadas en el control de la parasitosis y la condición económica de los ganaderos, lo cual conllevaría a inadecuados o ausentes tratamientos veterinarios y a una deficiente alimentación del ganado, dado que el estado nutricional influye en el poten- cial genético e inmunológico de los animales (Cordero del Campillo, 1999; Soca-Pérez et al., 2016).

El ganado alimentado con alfalfa y pastos naturales presentó una frecuencia de fascioliasis semejante a los que consumían estos alimentos más concentrado; de igual forma, la frecuencia fue semejante entre los que abrevaban diferentes fuentes de agua. Las similares proporciones y la ausencia de asociación se debe posiblemente a que el ganado está expuesto a prácticamente las mismas condiciones de alimentación y fuentes de agua, las cuales deben contener cargas parasitarias similares como consecuen- 
cia de la acumulación de la materia fecal, todo ello característico del sistema extensivo ganadero (Martín et al., 2001; Martínez et al., 2007).

En el Perú, son muy limitadas las investigaciones sobre prevalencias de $F$. hepatica en los hospederos intermediarios. Entre ellas, Larrea et al. (2007) reportan frecuencias de $17 \%$ en Lymnaea columella y L. viatrix procedentes de Cajamarca, Siancas (2017) en La Libertad encontró una frecuencia de $30.7 \%$ en L. viatrix y Londoñe et al. (2009) en Cusco encontraron infecciones en $P$. columella, que variaron entre 36 y $48 \%$. El presente estudio estimó una baja presencia de Fasciola en los Lymnaeidae del distrito de Huanca; sin embargo, se debe considerar el elevado potencial biótico de estos moluscos y de $F$. hepatica, dado que cada caracol puede producir 25000 nuevos individuos en tres meses y de cada miracidio del parásito se pueden generar 250 cercarias, de allí que no se debe subestimar las pequeñas cifras de caracoles infectados (Carrada-Bravo, 2007).

En la comunidad de San Basilio no se encontraron ejemplares Lymnaeidae, posiblemente debido a las condiciones medioambientales que estarían limitando su distribución y abundancia; por ello la importancia de contar con estudios ambientales y su influencia en el ciclo biológico de los caracoles. En este sentido, Pujadas et al. (2015) en Santa Fe, Argentina, describieron en su estudio las características fisicoquímicas de temperatu$\mathrm{ra}\left(20.6-29^{\circ} \mathrm{C}\right)$, conductividad $(0.6-2.3 \mathrm{mS} / \mathrm{cm})$ y $\mathrm{pH}$ ligeramente alcalino (7.6-8.3). Asimismo, Iturbe y Muñiz (2012) en su trabajo en Cusco, Perú, determinaron la calidad fisicoquímica de las aguas de los biotopos de los caracoles.

Las características conquiológicas de otros Lymnaeidae han sido reportados. Así, especímenes de Galba cubensis procedentes del Delta del Ebro en España, presentaron conchillas con dimensiones levemente mayores (alto: $8.1 \mathrm{~mm}$; ancho: $4.7 \mathrm{~mm}$ ) pero una altura menor $(3.8 \mathrm{~mm})$ (Schniebs et al.,
2018); caracoles Galba neotropica de Santa Fe, Argentina, mostraron longitudes de conchillas entre 4.1 y $8.5 \mathrm{~mm}$ y ancho entre 2.5 y $5.1 \mathrm{~mm}$ (Pujadas et al., 2015); asimismo, especímenes de Lymnaea neotropica y L. viator en Catamarca, Argentina, presentaron medidas superiores, además de tener peristoma delgado y ápice de la espira obtuso (Bargues et al., 2016), en tanto que para L. schirazensis se han reportado proporciones similares a las del presente estudio (Bargues et al., 2011). Investigaciones en Perú realizadas por Iturbe y Muñiz (2012) en San Jerónimo, Cusco, han descrito las conchillas de Galba truncatula dimensiones semejantes a los de la Zona del presente estudio (largo: $5.08 \mathrm{~mm}$, ancho: $2.85 \mathrm{~mm}$, ancho: $1.74 \mathrm{~mm}$, largo de la abertura: $2.64 \mathrm{~mm}$ ) aunque con mayor longitud para el largo de la espira $(2.6 \mathrm{~mm})$. Contrariamente, Londoñe et al. (2009) reportaron para Lymnaea viatrix largo y ancho de 4.5 y $1.1 \mathrm{~mm}$, respectivamente, y $0.7 \mathrm{~mm}$ de largo de abertura, siendo valores muy inferiores a las de esta investigación.

Respecto a la identificación taxonómica de los Lymnaeidae, algunos estudios enfocados en relacionar los rasgos conquiológicos con su posible dependencia genética, han demostrado que la morfología de las conchillas responde a las condiciones ambientales como consecuencia de su plasticidad fenotípica y no está determinada por la genética de estos caracoles (Wullschleger y Jokela, 2002). También se ha encontrado gran variabilidad en la morfología del aparato reproductor entre especies, pero mostrando incongruencia entre la caracterización conquiológica con los resultados genéticos. No obstante, Samadi et al. (2000) pudieron diferenciar L. cubensis de $L$. truncatula procedentes del viejo mundo y de países latinoamericanos como Bolivia mediante parámetros anatómicos.

Dadas las posibles confusiones para identificar especies de Lymnaeidae basándose en la descripción conquiológica, se ha utilizado pruebas moleculares para diferenciar especies fenotípicamente similares (Correa et 
al., 2011), utilizando secuencias de los nucleótidos ITS-2 e ITS-1 del ADN ribosómico (rDNA) y COI del ADN mitocondrial (mtDNA) (Hurtrez-Boussès et al., 2005; Bargues et al., 2007; 2012). No obstante, se considera que la información obtenida mediante la caracterización conquiológica de los Lymnaeidae es de utilidad en la determinación preliminar de la posición taxonómica, fundamental para el reconocimiento de estos especímenes en la etapa de campo y laboratorio.

\section{Conclusiones}

- La frecuencia de infección de Fasciola hepatica en el ganado del distrito de Huanca de la provincia de Caylloma, Arequipa en 2018, fue del 46.4\%.

- La variable especie pecuaria se asoció a la fascioliasis en el ganado, siendo los ovinos y equinos, los grupos con mayor probabilidad a desarrollar la enfermedad.

- La frecuencia de infección de Fasciola hepatica en caracoles Lymnaeidae fue $7.4 \%$.

\section{Agradecimiento}

Los autores agradecen a la Universidad Nacional de San Agustín de Arequipa por la financiación de la presente investigación con Contrato de Subvención N. ${ }^{\circ}$ TP-0342018-UNSA «Tesis de pregrado» convocatoria 2017, Perú. De igual forma, expresamos nuestra gratitud al Dr. A. Whittembury, Universidad Nacional Mayor de San Marcos, por su apoyo en el análisis de datos.

\section{Literatura Citada}

1. Acuña JA, Domínguez AH, Toro EM. 2012. Una comparación entre métodos estadísticos clásicos y técnicas metaheurísticas en el modelamiento estadístico. Scientia et Technica 50: 68-77.
2. Alpízar CE, Bianque de Oliveira J, Jiménez AE, Hernández J, Berrocal A, Romero JJ. 2013. Fasciola hepatica en ganado bovino de carne en Siquirres y lesiones anatomo-histopatológicas de hígados bovinos decomisados en mataderos de Costa Rica. Agron Costarric 37: 7-16.

3. Alzamora-Gonzales L, Echevarria RJ, Colona-Vallejos EH, Aguilar-Luis MA, de Amat-Herbozo CC. 2016. Desarrollo de ELISA sándwich indirecto para la determinación de antígenos de excreción-secreción de Fasciola hepática. Rev Peru Biol 23: 47-52. doi: 10.15381/rpb.v23i1.11833

4. Bargues MD, Artigas $P$, Mera $R L, Y$ Sierra, Pointier JP, Mas-Coma S. 2007. Characterisation of Lymnaea cubensis, $L$. viatrix and $L$. neotropica n. sp., the main vectors of Fasciola hepatica in Latin America, by analysis of their ribosomal and mitochondrial DNA. Ann Trop Med Parasit 101: 621641. doi: 10.1179/136485907X229077

5. Bargues MD, Artigas P, Khoubbane $M$, Flores R, Glöer P, Rojas-García $R$, Ashrafi K, et al. 2011. Lymnaea schirazensis an overlooked snail distorting fascioliasis data: genotype, phenotype, ecology, worldwide spread, susceptibility, applicability. PLoS One 6: e24567. doi: 10.1371/journal.pone.0024567

6. Bargues MD, Artigas P, Khoubbane M, Ortiz P, Naquira C, Mas-Coma S. 2012. Molecular characterisation of Galba truncatula, Lymnaea neotropica and L. schirazensis from Cajamarca, Perú and their potential role in transmission of human and animal fascioliasis. Parasites \& Vectors 5: 174. doi: 10.1186/1756-3305-5-174

7. Bargues MD, Malandrini JB. Artigas P, Soria CC, Velásquez JN, Carnevale $S$, Mateo L, et al. 2016. Human fascioliasis endemic areas in Argentina: multigene characterisation of the lymnaeid vectors and climatic-enviro- 
nmental assessment of the transmission pattern. Parasites \& Vectors 9: 306. doi: 10.1186/s13071-016-1589-z

8. Bedoya J, Hurtado Y, Pérez J, Solano S, Úsuga V, Vanegas M, Gómez, C, López J, et al. 2012. Primer registro de focos de fascioliasis y paramfistomosis en bovinos doble propósito, Gómez Plata, Antioquia, Colombia. Hechos Microbiol 3: 31-39.

9. Beltrán M, Otárola J, Tarqui K. 2014. Manual de procedimientos de laboratorio para el diagnóstico de los parásitos intestinales del hombre. Lima, Perú: Instituto Nacional de Salud. 66 p.

10. Berthe N, Tefera Y, Tintagu T, Muleta $W$. 2017. Small ruminant fascioliasis and its direct financial loss in Dessie municipal abattoir north eastern Ethiopia. J Vet Sci Technol 8: 490. doi: 10.4172/21577579.1000490.

11. Carrada-Bravo T. 2007. Fasciola hepatica: ciclo biológico y potencial biótico. Rev Mex Patol Clin 54: 21-27.

12. Carrada-Bravo T, Escamilla J. 2005. Fascioliasis: revisión clínico-epidemiológica actualizada. Rev Mex Patol Clin 52: 83-96.

13. Carvalho OS, Jannotti LK, Furtado CL, Marques PC, Lima R. 2014. Moluscos brasileiros de importância médica. $2^{\mathrm{a}}$ ed. Brasil: Belo Horizonte. $92 \mathrm{p}$.

14. Celis de la Rosa A de J, Labrada V. 2014. Bioestadística. $3^{\circ}$ ed. México: Manual Moderno. $813 \mathrm{p}$.

15. Cerda J, Vera C, Rada G 2013. Odds ratio: aspectos teóricos y prácticos. Rev Med Chile 141: 1329-1335. 10.4067/ S0034-98872013001000014

16. Cordero del Campillo M, RojoVásquez FD, Martínez AR, Sánchez MC, Hernández S, Navarrete I, Diez $P$, et al. 1999. Parasitología veterinaria. España: McGraw Hill Interamericana. $264 \mathrm{p}$.

17. Correa AC, Escobar JS, Noya O, Velásque LE, González-Ramírez C, Hurtrez-Boussès S, Pointier JP. 2011. Morphological and molecular characterization of Neotropic Lymnaeidae (Gas- tropoda: Lymnaeo-idea), vectors of fascioliasis. Infect Genet Evol 11: 19781988. doi: 10.1016/j.meegid.2011.09.003

18. Dagnino J. 2014. Intervalos de confianza. Rev Chil Anest 43: 129-133. doi: 10.25237/revchilanestv43n02.11

19. Damiani AC, Álvarez GF. 2005. Determinación de un patrón de fractura intencional en gasterópodos marinos (Golfo de San Matías, provincia de Río Negro). Relaciones de la Sociedad Argentina de Antropología 30: 277-295.

20. Dar Y, Amer S, Zein R, Dreyfuss G. 2016. Characterisation of Pseudosuccinea columella and Radix natalensis (Gastropoda: Lymnaeidae) in Egypt using shell and molecular data. Molluscan Res 36: 22-28. doi: 10.1080/13235818.2015.1064512

21. De Lucía M, Gutiérrez DE. 2017. Redescripción de Potamolithus supersulcatus Pilsbry, 1896 (Gastropoda, Tateidae) del sur de la cuenca Del Plata. Pap Avulsos Zool 57: 207-219. doi: http://dx.doi.org/10.11606/0031 1049.2017.57.17

22. Espinoza JR, Terashima A, HerreraVelit P, Marcos, LA. 2010. Fascioliasis humana y animal en el Perú: impacto en la economía de las zonas endémicas. Rev Peru Med Exp Salud Pública 27: 604-612.

23. [GERESA] Gerencia Regional de Salud, Arequipa. 2014. Análisis de situación de salud. Perú. $159 \mathrm{p}$.

24. Gebeye B, Keffale M, Adugna S. 2017. The prevalence and financial significance of ovine fascioliasis at Debre Berhan municipal abattoir, North Showa, Ethiopia. Middle-East Appl Sci 3: 1-7. doi: 10.5829/idosi.mejas.2017.-01.07

25. Giménez T, Núñez A, Chamorro N, Alarcón G. 2014. Estudio de la infección natural por Fasciola hepatica en Lymnaea spp en el distrito de Yabebyry, departamento de misiones, Paraguay. Comp Cienc Vet 4: 14-18.

26. Girão ES, Ueno H. 1985. Técnica de quatro tamises para o diagnóstico coprológico quantitativo da fasciolose dos ruminantes. Pesq Agropec Bras 20: 905-912. 
27. Giraldo JC, Díaz AM, Pulido MO. 2016. Prevalencia de Fasciola hepatica en bovinos sacrificados en la 'planta de beneficio del Municipio de Une, Cundinamarca, Colombia. Rev Inv Vet Perú 27: 751-757. doi: 10.15381/ rivep.v27i4.12572

28. Gomez-Puerta LA, Angulo-Tisoc JM, Pacheco JI, Lopez-Urbina MT, Gonzales $\boldsymbol{A E}$. 2019. Infección natural por Fasciola hepatica en cérvidos del Perú. Rev Peru Biol 26: 143-148. doi: 10.15381/rpb.v26i1.15918

29. Hurtrez-Boussès S, Pendino A, Barnabé C, Durand P, Rondelaud D, Durand C, Meunier C, et al. 2005. Comparison between shell morphology and genetic diversity in two sympatric lymnaeid snails, vectors of fasciolosis. Can J Zool 83: 1643-1648. doi: 10.1139/ Z05-150

30. Iturbe P, Muñiz F. 2012. Galba truncatula induced to infection with miracidia of Fasciola hepatica, collected in Huayllapampa, San Jerónimo, Cusco, Perú. Neotrop Helminthol 6: 211-217.

31. Jaja IF, Mushonga B, Grees E, Muchenje V. 2017. Financial loss estimation of bovine fascioliasis in slaughtered cattle in South Africa. Parasite Epidemiol Control 2: 27-34. doi: 10.1016/j.parepi.2017.10.001

32. Jara C, Escalante H, Cassana W, Davelois K, Benites A. 2018. Prevalencia de fascioliasis en ovinos y bovinos en la provincia de Pataz, La Libertad, Perú, mediante examen coproparasitológico y Western Blot. Rev Inv Vet Perú 29: 1421-1429. doi: 10.15381/ rivep.v29i4.15198

33. Larrea H, Flórez, M, Vivar R, Huamán P, Velásquez J. 2007. Hospederos intermediarios de Fasciola hepatica en el Perú. Rev Horizonte Méd 7: 39-46.

34. Liba JW, Atsanda NN, Francis MI. 2017. Economic loss from liver condemnation due to fascioliasis in slaughtered ruminants in Maiduguri abattoir, Borno State, Nigeria. J Adv Vet Anim Res 4: 65-70. doi: 10.5455 / javar.2017.d192
35. Londoñe P, Chávez A, Li O, Suárez F, Pezo D. 2009. Presencia de caracoles Lymnaeidae con formas larvarias de Fasciola hepatica en altitudes sobre los 4000 msnm en la sierra sur del Perú. Rev Inv Vet Perú 20: 58-65.

36. López LP, Romero J, Velásquez LE. 2008. Aislamiento de Paramphistomidae en vacas de leche y en el hospedador intermediario (Lymnaea truncatula y Lymnaea columella) en una granja del trópico alto en el occidente de Colombia. Rev Colomb Cienc Pec 21: 9-18.

37. López-Villacís IC, Artieda-Rojas JR, Mera-Andrade RI, Muñoz-Espinoza MS, Rivera-Guerra VE, CuadradoGuevara AC, Zurita-Vásquez JH, et al. 2017. Fasciola hepatica: aspectos relevantes en la salud animal. J Andina Anim Sci 4: 137-146.

38. Martín M, Sánchez E, Mesías FJ, Rodríguez de Ledesma A, Pulido F. 2001. Sistemas extensivos de producción animal. Arch Zootec 50: 465-489.

39. Martínez LM, Gerritsen PRW, Blanco CV, Cárdenas OG, Contreras $S$, Cuevas R, Esparza JP, et al. 2007. Estado actual y perspectivas de la ganadería extensiva en la Sierra de Manantlán, en el occidente de México. México: Petra Ed. 73 p.

40. Meaza M, Keda A, Serda B, Sulayman M. 2017. Prevalence, gross pathological lesions and financial losses of bovine fascioliasis in Arba Minch Municipal abattoir, Gamo Gofa Zone, Southern Ethiopia. J Vet Med Anim Health 9: 186192. doi: 10.5897/JVMAH2016.0523

41. Merino K, Valderrama AA. 2017. Fasciola hepatica en bovinos del valle interandino de Aymaraes (Perú): identificación de factores asociados. Red Med Vet 34: 137-147. doi: 10.19052/mv.4262

42. Milton JS. 2007. Estadística para biología y ciencias de la salud. $3_{-}^{\circ}$ ed. España: McGraw Hill Interamericana. 217 p.

43. Núñez V. 2011. Revisión de dos especies de Physidae. Rev Mex Biodiv 82: 93-108. doi: http://dx.doi.org/10.22201/ ib.20078706e.2011.1.1193 
44. Otzen T, Manterola C. 2017. Técnicas de muestreo sobre una población. Int J Morphol 35: 227-232. doi: 10.4067/ S0717-95022017000100037

45. Palacio D, Bertot JA, Beltrao M, Vázquez A, Ortíz RC, Varona M. 2019. Economic losses induced by Fasciola hepatica in cattle slaughtered in Chacuba slaughterhouse, Camagüey, Cuba. Cuban J Agr Sci 53: 35-39.

46. [PANAFTOSA] Centro Panamericano de Fiebre Aftosa y Salud Pública Veterinaria. 2017. Manual veterinario de toma y envío de muestras. Brasil: Horizonte Educação e Comunicação. 80 p.

47. Pujadas JM, Farber M, Pointier JP, Giudici C, Wisnivesky C, Prepelitchi L. 2015. New record for Galba neotropica (d'Orbigny, 1835) in Argentina, with a detailed analysis of its morphology and molecular characteristics. Molluscan Res 35: 153-160. doi: 10.1080/13235818.2015.1044925

48. Rojas D, Cartín JA. 2016. Prevalencia de Fasciola hepatica y pérdidas económicas asociadas al decomiso de hígados en tres mataderos de clase A de Costa Rica. Agron Costarric 40: 53-62. doi: 10.15517/rac.v40i2.27366

49. Samadi S, Roumegoux A, Bargues MD, Mas-Coma S, Yong M, Pointier JP. 2000. Morphological studies of Lymnaeid snails from the human Fascioliasis endemic zone of Bolivia. J Moll Stud 66: 31-44. doi: 10.1093/mollus/ 66.1.31

50. Schniebs K, Glöer P, QuiñomeroSalgado S, Lopez-Soriano J, Hundsdoerfer A. 2018. The first record of Galba cubensis (L. Pfeiffer, 1839) (Mollusca: Gastropoda: Lymnaeidae) from open fields of Europe. Folia Malacol 26: 3-15. doi: https://doi.org/10.12657/ folmal.026.002

51. Siancas F. 2017. Frecuencia de infección por Fasciola hepatica en Lymnaea viatrix e identificación de plantas asociadas en la provincia de Pataz, Región La Libertad-Perú, 2016. Tesis de Biólogo. Trujillo, Perú: Univ. Nacional de Trujillo. 12 p.
52. Soca-Pérez M, Giupponi-Cardoso P, López-Vigoa O, Sanavria A, SánchezSantana T, Labrada-Vázquez A. 2016. Prevalencia de Fasciola hepatica en vacas en pastoreo durante el periodo poco 1luvioso. Pastos y Forrajes 39: 281-285.

53. Ticona D, Chávez A, Casas G, Chavera A, Li O. 2010. Prevalencia de Fasciola hepatica en bovinos y ovinos de Vilcashuamán, Ayacucho. Rev Inv Vet Perú 21: 168-174.

54. Tomassi CA, Soria CC, Fresco MJ, Covarrubia E, Valdez, Malandrini JB. 2015. Técnicas de obtención de cuerpos de moluscos lymneidos para su identificación taxonómica. Rev Electr Iberoam Educ Cienc Tecnol 6: 51-65.

55. Trujillo CC. 2015. Análisis territorial del paisaje agrario del distrito de Huanca, provincia de Caylloma, región Arequipa. [Internet]. Disponible en: http:// www.descosur.org.pe/wp-content/ uploads/2016/06/Carlos-Trujillo.pdf

56. Trujillo CC, Lajo M. 2015. Análisis territorial del paisaje agrario del distrito de Huanca, provincia de Caylloma, región Arequipa. En: XI Congreso Nacional de Geografía. Cajamarca: Sociedad Geográfica de Lima.

57. Valderrama AA. 2016. Prevalencia de fascioliasis en animales poligástricos de Perú, 1985-2015. Rev Med Vet 2016: 121-129. doi: 10.19052/mv.3861

58. Vázquez AA, Sánchez J. 2015. Clave ilustrada y comentada para la identificación de moluscos gastrópodos fluviales de Cuba. Rev Cubana Med Trop 67: 231-243

59. Velásquez S. 2017. Prevalencia de distomatosis hepática (Fasciola hepatica) en equinos en el distrito de Socabaya, provincia de Arequipa, departamento de Arequipa-2016. Tesis de Médico Veterinario. Arequipa, Perú: Univ. Católica de Santa María. $56 \mathrm{p}$.

60. Wullschleger EB, Jokela J. 2002. Morphological plasticity and divergence in life-history traits between two closely related freshwater snails, Lymnaea ovata and Lymnaea peregra. J Moll Stud 68: 1-5. doi: 10.1093/mollus/68.1.1 